\title{
A Subcell Resolution Method for Viscous Systems of Conservation Laws
}

\author{
EdUARd HaRabetian* \\ Department of Mathematics, University of Michigan, Ann Arbor, Michigan 48109
}

Received August 27, 1990; revised January 10, 1992

\begin{abstract}
We consider the generalization of scalar subcell resolution schemes to systems of viscous conservation laws. For this purpose we use a weakly nonlinear geometrical optics approximation for parabolic perturbations of hyperbolic conservation laws and the Roe-type field by field decomposition. Computations of the reactive Navier-Stokes equations are presented as an application. (c) 1992 Academic Press, Inc.
\end{abstract}

\section{INTRODUCTION AND SUBCELL MODELS}

In this paper we consider numerical approximations to viscous perturbations of hyperbolic systems of the form

$$
u_{t}+f(u)_{x}=\varepsilon\left(B(u) u_{x}\right)_{x},
$$

where $f_{u}=A(u)$ has real eigenvalues $\lambda_{i}(u), i=1, \ldots, n$, and $B(u)$ is positive. These equations serve as a model for compressible viscous flow.

When $\varepsilon$ is small, solutions of (1) typically develop viscous shock layers where $u$ changes rapidly over very narrow zones. Sophisticated methods have been developed for the purely hyperbolic case $(\varepsilon=0)[13,21,20,23]$; these schemes capture shock discontinuities over very narrow zones without overshoots or undershoots. So far, numerical schemes which approximate (1) have relied on splitting the hyperbolic and parabolic parts and approximating the viscous term separately by the centered difference approximation. To avoid this splitting error, we are considering a numerical scheme that uses a subcell model for which one can obtain a time-accurate evolution. This scheme is designed to work on a coarse grid, i.e., where the viscous layers are not resolved. For scalar equations and convex flux functions $f$, such a numerical method was proposed in $[10,11]$. We now describe how this method was derived in terms of a subcell resolution model.

For simplicity let us assume that $B(u)=1$. Consider a

* Research supported by NSF Grant DMS-9003965. spatial grid with uniform spacing $h$ and cells $I_{j}=$ $\left[x_{j-1 / 1}, x_{j+1 / 2}\right]$. The solution is approximated at cell centers $u\left(x_{j}\right) \approx u_{j}$. A subcell function $g_{j+1 / 2}(x)$ is a function which is defined on the interval $I_{j+1 / 2}=\left[x_{j}, x_{j+1}\right]$ such that $g_{j+1 / 2}\left(x_{j}\right)=u_{j}$, and $g_{j+1 / 2}\left(x_{j+1}\right)=u_{j+1}$. Let us denote by $S(t)$ an approximate, or possibly exact, time evolution solver; i.e., $S(t) u(x)$ is the solution at time $t$ with the initial data at $t=0$ given by $u(x)$. The choices of $S$ and $g$ should be motivated by the fact that one would like to be able to easily compute $S(t) g_{j+1 / 2}(x)$. With these ingredients one can design a conservation form scheme with numerical flux $h_{j+1 / 2}$ which is consistent with the exact flux $f(u)-\varepsilon B(u) u_{x}$ :

$$
\begin{gathered}
\frac{u_{j}^{n+1}-u_{j}^{n}}{\Delta t}+\frac{h_{j+1 / 2}-h_{j-1 / 2}}{\Delta x}=0 \\
h_{j+1 / 2}=f\left(S\left(\frac{\Delta t}{2}\right) g_{j+1 / 2}\right)\left(x_{j+1 / 2}\right) \\
-\varepsilon\left(S\left(\frac{\Delta t}{2}\right) g_{j+1 / 2}\right)_{x}\left(x_{j+1 / 2}\right) .
\end{gathered}
$$

The choice of the subcell function $R$ should depend on the ratio of grid size $h$ to the viscosity $\varepsilon$. For example, if $h / \varepsilon \ll 1$, then the solution "looks" smooth on the given grid and the only reasonable choice of $g_{j+1 / 2}$ is the linear interpolant. Since the second derivative of a linear function is zero, it is now also reasonable to define an approximate solver $S$ that ignores the viscous term,

$$
S(t) g_{j+1 / 2}(x)=g_{j+1 / 2}\left(x-a_{j+1 / 2} t\right),
$$

where $a_{j+1 / 2}$ is the approximate convective speed:

$$
a_{j+1 / 2}=\frac{f\left(u_{j+1}\right)-f\left(u_{j}\right)}{u_{j+1}-u_{j}}
$$

It is easy to see that this scheme consists of a Lax-Wendroff 
type approximation for the hyperbolic part and a centered difference approximation for the viscous part. The viscosity is not taken into account in the evolution operator. While this error should be small for "smooth" solutions (in fact the scheme is second order accurate), large errors due to oscillations should occur when $h / \varepsilon$ is not small.

The next example is the other extreme case, i.e., when $h / \varepsilon \gg 1$. Here the only reasonable choice of $g_{j+1 / 2}$ is the step function with a jump at $x_{j+1 / 2}$. For $S$ we can choose the exact solution operator with $\varepsilon=0$. The resulting scheme is the Godunov scheme for the hyperbolic equation with zero viscosity which is first-order accurate but it is monotone and hence total variation diminishing.

Finally, when

$$
h / \varepsilon \approx 1
$$

one could consider more complicated choices of a subcell function. In [10] we proposed that $g_{j+1 / 2}$ be the travelling wave solution of (1). Consider only the case $u_{j}>u_{j+1}$ (shock) and the travelling wave solutions (a.k.a. viscous profiles) that interpolate the data between the two grid points $x_{j}, x_{j+1}$, i.e., solutions of the form: $u(t, x)=$ $\phi(x-s t)$, where $\phi$ must satisfy the following sccond-order ordinary differential equation

$$
\varepsilon \phi^{\prime \prime}=-s \phi^{\prime}+f(\phi)^{\prime}
$$

with the following boundary conditions:

$$
\phi\left(x_{j}\right)=u_{j}, \quad \phi\left(x_{j+1}\right)=u_{j+1} .
$$

To determine the unknown speed $s$, one additional condition must be imposed; if $f$ is an even function (e.g., Burgers equation, $f(u)=u^{2}$ ), it is reasonable to impose the condition that the profile is symmetric,

$$
\phi\left(x_{j, 1 / 2}+x\right)-\hat{u}=-\left(\phi\left(x_{j+1 / 2}-x\right)-\hat{u}\right),
$$

where $\hat{u}$ is the average of $u_{j}, u_{j+1}$. The viscous profile $\phi$ is determined uniquely; in the case of the Burgers equation, an explicit formula is available. The solver $S$ is defined as follows:

$$
S(t) g_{j+1 / 2}=g_{j+1 / 2}(x-s t) .
$$

We make two remarks:

1. The subcell function depends on the ratio $h / \varepsilon$ and tends to the linear subcell function when this ratio goes to zero, and it tends to the step function when this ratio gocs to infinity.

2. The solver $S(t)$ is an exact solver, since $\phi$ is a travelling wave solution.
One can show that this scheme is second-order accurate (formally) in the sense that

$$
\left|h_{j+1 / 2}-\left(f(u)-\varepsilon u_{x}\right)\left(x_{j+1 / 2}, \frac{\Delta t}{2}\right)\right| \leqslant C(h / \varepsilon) h^{2},
$$

where $u$ is the exact smooth solution which interpolates $u_{j}, u_{j+1}$ at time zero. The constant $C(h / \varepsilon)$ depends (in addition to $h / \varepsilon$ ) only on derivatives up to second order of the solution at time zero.

One can show this scheme is TVD when parameters $h / \varepsilon$ and $\Delta t / h$ belong to a certain region in the parameter plane. In addition, in order for a three-point scheme to be both second-order accurate and TVD diminishing, these parameters must belong to another (slightly larger) region of the plane. The proof of these are in [10].

In this paper we would like to extend these ideas to systems. Our goal is to construct a good approximate solution at the subcell level that can be used in the computation of the viscous flux at the cell boundary. In Section 2 we show how to construct such a subcell approximation from a superposition of scalar viscous traveling waves. In the Appendix, we will show that the approximation is formally valid in the weakly nonlinear geometrical optics asymptotic regime $[14,19]$. This approximation is supposed to work for weak viscous shocks. However, in practice strong viscous shocks are also well approximated. Next, we show how to modify the scheme for practical purposes and point out the connection with flux-limiting TVD schemes $[13,23]$. Finally, in the last section we show some computations for reactive, compressible flows in one dimension. These computations are on coarse grids and compare favorably in terms of resolution with other computations that use standard schemes (cf. [3]).

In conclusion to this introduction, we mention that Harten [12] has introduced a different subcell resolution idea in connection with reconstructing discontinuities for sharp shock capturing in 1D and Engquist and Sjogreen [8] have used idcas closely related to Harten's to solve stiff problems with source terms, such as inviscid combustion.

\section{THE SUBCELL APPROXIMATION FOR SYSTEMS}

In this section we show how to construct a subcell approximation to (1) using the weakly nonlinear geometrical optics approximation which has been developed by several authors $[14,19]$ and propose a numerical method based on such an approximation. We also discuss practical modifications and point out connections between our scheme and the TVD flux-limiting schemes from $[13,23]$ for systems of hyperbolic conservation laws. 
We start with the Roe decomposition,

$$
u_{j+1}-u_{j}=\sum_{k} b_{k} R_{k}\left(u_{0}\right)
$$

where $u_{0}$ is the Roe average and $R_{k}$ 's are the cigenvectors of $A\left(u_{0}\right)=f^{\prime}\left(u_{0}\right)$. The Roe average $u_{0}$ satisfies $A\left(u_{0}\right)\left(u_{j+1}-u_{j}\right)=f\left(u_{j+1}\right)-f\left(u_{j}\right)$ and has nicer properties than the arithmetic average of $u_{j}$ and $u_{j+1}$ [21].

In the interval $I_{j+1 / 2}=\left[x_{j}, x_{j+1}\right]$, we consider the ansatz

$$
\begin{aligned}
u(t, x) & =u_{0}-\frac{1}{2} \sum_{k} b_{k} \phi_{k} R_{k}+\cdots \\
& =u_{0}-\frac{1}{2} u_{1}(t, x)+\cdots, \\
\phi_{k} & =\phi_{k}\left(\frac{x-\lambda_{k} t}{\varepsilon / b_{k}}\right),
\end{aligned}
$$

where $\lambda_{k}$ 's are the eigenvalues of $A\left(u_{0}\right), b_{k}$ and, hence, $u_{1}$ are small and $\phi_{k}$ 's will be determined later. We consider only genuinely nonlinear fields. Upon substituting the ansatz into Eq. (1) and throwing away lower order terms in $u_{1}$, one obtains viscous travelling wave solutions to Burgers equation:

$$
\phi_{k}=\frac{1}{\alpha_{k}} \tanh \left(\frac{x-x_{j+1 / 2}-\lambda_{k} t}{4 \alpha_{k} \varepsilon / b_{k}}\right) .
$$

This calculation is the same as the weakly nonlinear geometrical optics calculation in $[14,19]$ and we have included the details in the Appendix. The approximate solution is then a superposition of travelling waves (appendix equation (25)),

$$
\begin{aligned}
u(x, t)= & u_{0}-\frac{1}{2} \sum_{k} b_{k} \frac{1}{\alpha_{k}} \\
& \times \tanh \left(\frac{x-x_{j+1 / 2}-\lambda_{k} t}{4 \alpha_{k} \varepsilon / b_{k}}\right) R_{k}, \quad x \in I_{j+1 / 2} .
\end{aligned}
$$

Moreover, the constants $\alpha_{k}$ are uniquely determined by the boundary conditions $u\left(x_{j}, 0\right)=u_{j}$ and $u\left(x_{j+1}, 0\right)=u_{j+1}$ or

$$
\phi\left(\frac{x_{j}}{\varepsilon / b_{k}}\right)=1, \quad \phi\left(\frac{x_{j+1}}{\varepsilon / b_{k}}\right)=-1
$$

if $u_{0}$ is the arithmetic average. Since the $\phi_{k}$ 's are symmetric with respect to the center of the interval $I_{j+1 / 2}$, both conditions in (5) are satisfied if

$$
\frac{1}{\alpha_{k}} \tanh \left(\frac{h h_{k}}{8 \alpha_{k} \varepsilon}\right)=-1
$$

Note that (6) does not have a real solution $\alpha_{k}$ unless $b_{k} \leqslant 0$. For scalar equations this means that $u_{j+1} \leqslant u_{j}$ (shock). This suggests that we should treat the system as if there was a shock in every field and solve for $\alpha_{k}$ by modifying Eq. (6) as

$$
\frac{1}{\alpha_{k}} \tanh \left(\frac{h\left|b_{k}\right|}{8 \alpha_{k} \varepsilon}\right)=1
$$

This completes the description of the subcell model. We now turn to the numerical method.

One could use the following formula for the numerical flux (see (2)),

$$
f\left(u\left(x_{j+1 / 2}, \Delta t / 2\right)\right)-\varepsilon B\left(u\left(x_{j+1 / 2}, \Delta t / 2\right)\right) u_{x}\left(x_{j+1 / 2}, \Delta t / 2\right),
$$

where $u(x, t)$ is the subcell approximation which is given in (4). However, since $u=u_{0}-\frac{1}{2} u_{1}(3)$, we use the expansion

$$
f\left(u\left(x_{i+1 / 2}, \Delta t / 2\right)\right)=f\left(u_{0}\right)-\frac{1}{2} A_{0} u_{1}\left(x_{j+1 / 2}, \Delta t / 2\right)+\cdots
$$

and a similar one for $B$, to define a numerical flux as

$$
\begin{aligned}
h_{j+1 / 2}= & f\left(u_{0}\right)-\frac{1}{2} A_{0} u_{1}\left(x_{j+1 / 2}, \Delta t / 2\right) \\
& -\varepsilon B_{0} u_{1_{x}}\left(x_{j+1 / 2}, \Delta t / 2\right) .
\end{aligned}
$$

Remark. The terms discarded here are of the same order in $u_{1}$ as the terms discarded in the weakly nonlinear approximation (4).

We shall write the numerical flux (8) in a form which reveals its relation to flux limiters and TVD schemes $[13,23]$. Let us define

$$
s_{k}=h\left|h_{k}\right| / 8 \varepsilon \alpha_{k} .
$$

Then $s_{k}$ is a "smoothness" parameter, in the sense that it is small when the solution is "smooth" in the $k$ th field (i.e., $b_{k}$ is small).

Remark. If $h \approx e$ and $b_{k}$ is small then from (7) $\alpha_{k}=\sqrt{h\left|b_{k}\right| / 8 \varepsilon}+\cdots$ so $s_{k}=\sqrt{h\left|b_{k}\right| / 8 \varepsilon}+\cdots$.

Since $\alpha_{k}=\tanh \left(s_{k}\right)$ (cf. (7)), and since, as we did beforc, we suppose that $b_{k} \leqslant 0$, it follows from (4) that

$$
\begin{aligned}
u_{1}(x, t)= & \sum_{k} b_{k} \frac{1}{\tanh \left(s_{k}\right)} \\
& \times \tanh \left(-2 s_{k} \frac{x-x_{j+1 / 2}-\lambda_{k} t}{h}\right) R_{k} .
\end{aligned}
$$


Using this formula for $u_{1}$, after a small calculation, it follows from (8) that

$$
h_{j+1 / 2}=f\left(u_{0}\right)-\frac{1}{2} \sum_{k}\left(\varphi_{k}^{s_{k}}\left(c_{k}\right) \lambda_{k}+\frac{2 \varepsilon}{h} B_{0}\left(\varphi_{k}^{s_{k}}\right)^{\prime}\left(c_{k}\right)\right) b_{k} R_{k},
$$

where

$$
\varphi_{k}^{s k}\left(c_{k}\right)=\frac{\tanh \left(c_{k} s_{k}\right)}{\tanh \left(s_{k}\right)}, \quad c_{k}=\frac{\lambda_{k} \Delta t}{h}
$$

The first sum in the numcrical flux is the artificial viscosity, the second sum is the real viscosity. The balance between the two is achieved as follows: The artificial viscosity is minimized in smooth regions and maximized inside shock layers. The opposite effect occurs with the real viscosity. The parameter $s_{k}$ serves as a detector of "nonsmooth" regions and, depending on its size, the subcell scheme tends to the centered-Lax-Wendroff scheme in one case and to the Roe scheme in the other case as the subcell function gradually changes from a linear function to a step function.

In practice, this scheme is unfortunately not robust enough since, in order to suppress oscillations, one has to find the appropriate value of $h / \varepsilon$ (cf. [10]), and for systems this can only be done with limited success. In addition, many evaluations of tanh are required which slow down the computation considerably. With very minor modifications, however, we can recover the robustness of a TVD scheme. The only modifications are to replace $\varphi_{k}^{s_{k}}$ by a piecewise linear approximation and to choose a different smoothness parameter to replace $s_{k}$.

In the TVD approach, as described, for example, in [23], the measure of smoothness is given by the parameters

$$
\begin{gathered}
r_{k, j}^{+}=\frac{c_{k, j-1}^{+}\left(1-c_{k, j-1}^{+}\right) b_{k, j-1}}{c_{k, j}^{+}\left(1-c_{k, j}^{+}\right) b_{k, j}} \\
r_{k, j+1}^{-}=\frac{c_{k, j+1}^{-}\left(1+c_{k, j+1}^{-}\right) b_{k, j+1}}{c_{k, j}^{-}\left(1+c_{k, j}^{-}\right) b_{k, j}},
\end{gathered}
$$

where the subscripts $j-1, j, j+1$ refer to the quantities computed in the intervals $I_{j-1 / 2}, I_{j+1 / 2}, I_{j+3 / 2}$, respectively, and the superscripts,+- denote the positive and negative parts, respectively. We refer the reader to $[13,23]$ for the details as to what motivates this choice. Let us define

$$
\begin{aligned}
s_{k, j}^{+} & =1-r_{k, j}^{+} \\
s_{k, j+1}^{-} & =1-r_{k, j+1}^{-}
\end{aligned}
$$

and note that $s_{k, j}^{+}, s_{k, j+1}^{-}$tend to 0 when the solution is smooth. The limiter $\varphi_{k}^{s_{k}}$ in (12) is approximated now by a piecewise linear function as follows:

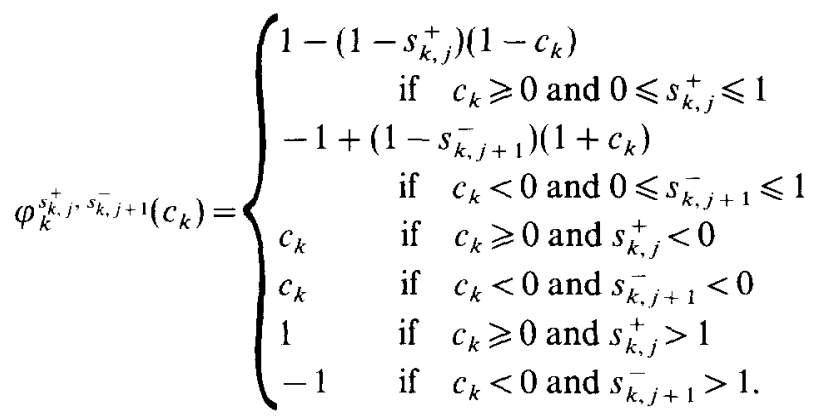
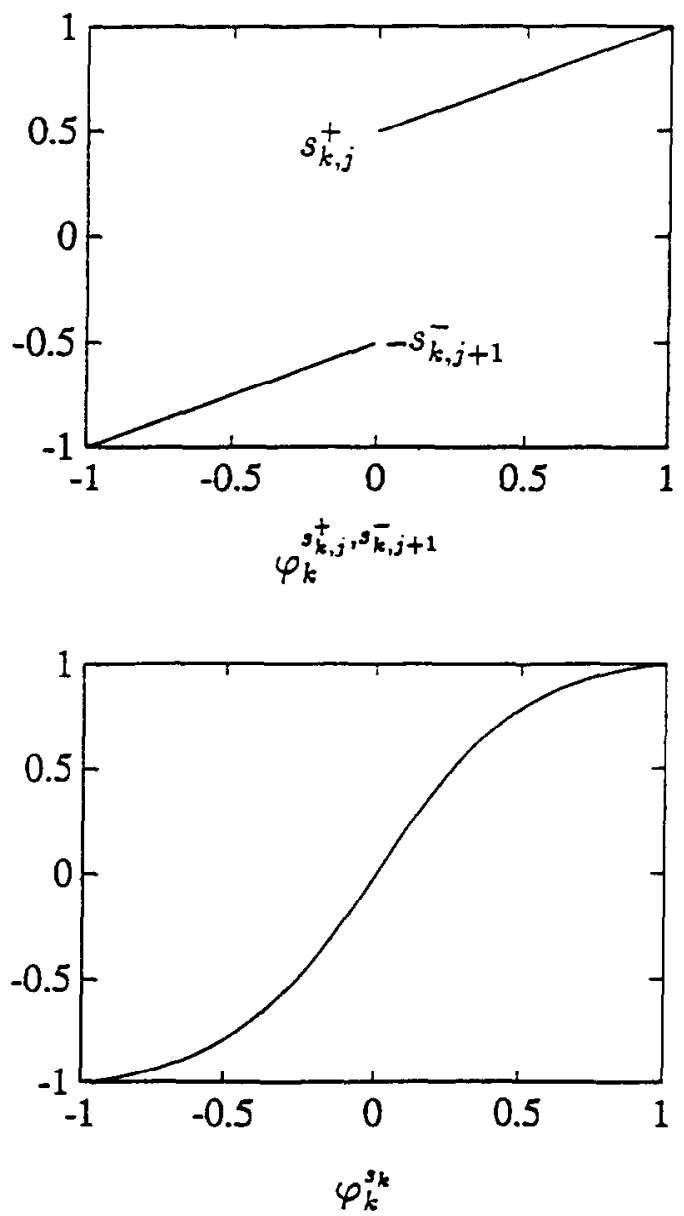

We now replace $\varphi_{k}^{s_{k}}$ in (11) by $\varphi_{k}^{s_{k, j}^{+}, s_{k, j+1}^{-}}$above; since the function is discontinuous (piecewise linear), the derivative in (11) is taken from only one side. This completes the description of the scheme.

Without the $\varepsilon$-term, i.e., the real viscosity term, the numerical flux (11) is a TVD flux as described in [23] for hyperbolic conservation laws. This is a different interpretation of flux-limiters, one that is based on subcell resolution models. 


\section{COMPUTATIONAL RESULTS FOR AN APPLICATION}

In this section we apply the subcell resolution scheme to the computation of particular solutions of the $1 D$ reactive and compressible Navier-Stokes equations. These problems are characterized by the presence of different phenomena occuring on different scales such as sound (shock) propagation, diffusion, and reaction. Our goal is to study the effects of viscosity on the structure of combustion waves. The subcell method combines the propagation and diffusion without any splitting. The reaction term is a lower order source term in the equations and is treated here by a standard implicit trapezoidal method so it is not part at the subcell model. In our computations we will consider moderate reaction rates, in particular, not large compared to other parameters. Inviscid problems with stiff terms have been solved, for example, by Engquist and Sjogreen by a robust subcell method [8].

According to Von Neumann [4], in the presence of viscosity and a finite (not large) reaction rate the structure of a detonation wave is given by a slow deflagration (flame) across which the pressure remains constant and is initiated by a fluid mechanical shock. This precursor shock is responsible for the rise in temperature that initiates the flame. The speed of the flame changes with the amount of viscosity present.

When the reaction rate is raised to a critical level the detonation wave reappears but bifurcations may occur when the viscosity in the problem is changed. This last statement has been analyzed rigorously by A. Majda for a simple $2 \times 2$ model system of equations [18]. The bifurcation occurs from a $\mathrm{CJ}$ detonation characterized by a sharp pressure spike to a faster moving weak detonation.

The reactive, compressible Navier-Stokes equations are

$$
\begin{aligned}
\rho_{t}+(\rho u)_{x} & =0 \\
(\rho u)_{t}+\left(\rho u^{2}+p\right)_{x} & =\mu u_{x x} \\
(\rho E)_{t}+(\rho u E+u p)_{x} & =\mu\left(\frac{u^{2}}{2}\right)_{x x}+\left(q_{0} \rho d Z_{x}\right)_{x}+c_{p} \lambda T_{x x} \\
(\rho Z)_{t}+(\rho u Z)_{x} & =-S(T) \rho Z+\left(d \rho Z_{x}\right)_{x},
\end{aligned}
$$

where (cf. $[9,6]$ )

$\rho, u, p, e, T=$ density, velocity, pressure, internal energy, temperature

$\mu, \lambda, d=$ coefficients of viscosity, heat conduction, and species diffusion

$Z=m_{f} /\left(m_{f}+m_{b}\right), m_{f}, m_{b}=$ mass of fresh, burnt gas

$c_{v}, c_{p}=$ coefficients of specific heat at constant volume, pressure
$E=e+u^{2} / 2+q_{0} Z$ is the total energy, $q_{0}=$ heat release (positive for exothermic reactions)

$e=(1 /(\gamma-1) \rho)(p / p)=c_{v} T$ is the polytropic gas law and $\gamma=c_{p} / c_{v}$.

For $S(T)$ we use a simple model given by the ignition type kinetics [3],

$$
S(T)=K_{0} H\left(T-T_{*}\right)
$$

where $H$ is the Heaviside function and $K_{0}$ is a large constant that measures the reaction rate. The parameter that measures the stiffness in the numerical problem is, however, given by $K_{0} \Delta t$.

To simplify things we will assume that

$$
\mu=\gamma \lambda=d
$$

This implies that there is only one viscous scale in the problem given by $\mu$. The more general situation, when, for example, $\mu$ and $\lambda$ are allowed to be sufficiently different from each other in magnitude, is of great physical importance but unfortunately does not fall withing the framework developed here.

In order to compute the numerical flux, we should write the viscosity terms from (14) in terms of the conserved variables $U=(\rho, \rho u, \rho E, \rho Z)$. Using (16), a brief calculation yields

$$
\begin{gathered}
\mu u_{x x}=\mu\left(\frac{(\rho u)_{x}-u p_{x}}{\rho}\right)_{x} \\
\mu\left(\frac{u^{2}}{2}\right)_{x x}+\left(q_{0} \rho d Z_{x}\right)_{x}+c_{p} \lambda T_{x x} \\
=\mu\left(-\frac{\rho E+q_{0}(\rho-1) \rho Z}{\rho^{2}} \rho_{x}+\frac{1}{\rho}(\rho E)_{x}\right. \\
\left.+\frac{q_{0}(\rho-1)}{\rho}(\rho Z)_{x}\right)_{x} \\
\left(d \rho Z_{x}\right)_{x}=\mu\left((\rho Z)_{x}-Z \rho_{x}\right)_{x} .
\end{gathered}
$$

We now turn to the Roe decomposition for the hyperbolic part of the system (14) (cf. Section 2). We need to find a state $U_{0}$ with the property

$$
A\left(U_{0}\right) A U=f\left(U_{j+1}\right)-f\left(U_{j}\right)
$$

where $f(U)=\left(\rho u, \rho u^{2}+p, \rho u E+u p, \rho u Z\right)$ is the flux vector from (14) and $A(U)$ is its Jacobian derivative. By following 
Roe's calculation in the non-reactive case [21], one easily obtains the Roe averages

$$
\begin{aligned}
\rho_{0} & =\sqrt{\rho_{j} \rho_{j+1}} \\
u_{0} & =\frac{u_{j} \sqrt{\rho_{j}}+u_{j+1} \sqrt{\rho_{j+1}}}{\sqrt{\rho_{j}}+\sqrt{\rho_{j+1}}} \\
H_{0} & =\frac{H_{j} \sqrt{\rho_{j}}+H_{j+1} \sqrt{\rho_{j+1}}}{\sqrt{\rho_{j}}+\sqrt{\rho_{j+1}}} \\
Z_{0} & =\frac{Z_{j} \sqrt{\rho_{j}}+Z_{j+1} \sqrt{\rho_{j+1}}}{\sqrt{\rho_{j}}+\sqrt{\rho_{j+1}}},
\end{aligned}
$$

where the total enthalpy $H=\gamma e+u^{2} / 2$. From these averages one can compute $U_{0}$ and verify that (18) holds.

In order to be able to compute the numerical flux (11), we need to compute the eigenvalues and eigenvectors $\lambda_{k}, R_{k}, k=1, \ldots, 4$, of $A\left(U_{0}\right)$, and the coefficients $b_{k}$ from the decomposition $\Delta U=\sum_{k} b_{k} R_{k}$. We provided the result of these straightforward computations,

$$
\begin{aligned}
\hat{\lambda}_{1}=u_{0} & -c_{0}, \quad \lambda_{2}=u_{0}+c_{0}, \quad \lambda_{3}=u_{0}, \quad \lambda_{4}=u_{0} \\
R_{1} & =\left(1, u_{0}-c_{0}, H_{0}+q_{0}-u_{0} c_{0}, Z_{0}\right) \\
R_{2} & =\left(1, u_{0}+c_{0}, H_{0}+q_{0}+u_{0} c_{0}, Z_{0}\right) \\
R_{3} & =\left(1, u_{0}, \frac{u_{0}^{2}}{2}, 0\right) \\
R_{4} & =\left(0,0, q_{0}, 1\right) \\
b_{1} & =\frac{\eta_{1}-\eta_{2}}{2} \\
b_{2} & =\frac{\eta_{1}+\eta_{2}}{2} \\
b_{3} & =\Delta \rho-\eta_{1} \\
b_{4} & =\Delta(\rho Z)-\eta_{1} Z_{0},
\end{aligned}
$$

where

$$
\begin{aligned}
& \eta_{1}=\frac{\Delta(\rho E)-q_{0} \Delta(\rho Z)+\Delta p u_{0}^{2} / 2-u_{0} \Delta(\rho u)}{c_{0}^{2} /(\gamma-1)} \\
& \eta_{2}=\frac{\Delta(\rho u)-u_{0} \Delta p}{c_{0}}
\end{aligned}
$$

and the speed of sound $c$ is given by

$$
c=\sqrt{\gamma(p / \rho)}
$$

The computation of the numerical flux in (11) is now straightforward: The entries in the matrix $B_{0}$ are obtained from (17) and evaluated from the Roe averages (19). The limiters for the genuinely nonlinear fields 1 and 2 are given by (13); for the linearly degenerate field 3,4 , we use the superbee limiter (cf. [23]) which has the effect of sharpening contact discontinuities.

The numerical flux should be modified to take into account the reaction term in the last equation of (14). A simple second-order accurate method is the implicit trapezoidal method. In our case it can be easily applied to the last equation of (14) as follows:

$$
\begin{aligned}
(\rho Z)_{t}+S(T) \rho Z \approx & \frac{(\rho Z)^{n+1}-(\rho Z)^{n}}{\Delta t} \\
& +\frac{S\left(T^{n+1}\right)(\rho Z)^{n+1}+S\left(T^{n}\right)(\rho Z)^{n}}{2} .
\end{aligned}
$$

This, added to the numerical flux difference (11) gives a complete description of the scheme. Note that $T^{n+1}$ in (21)
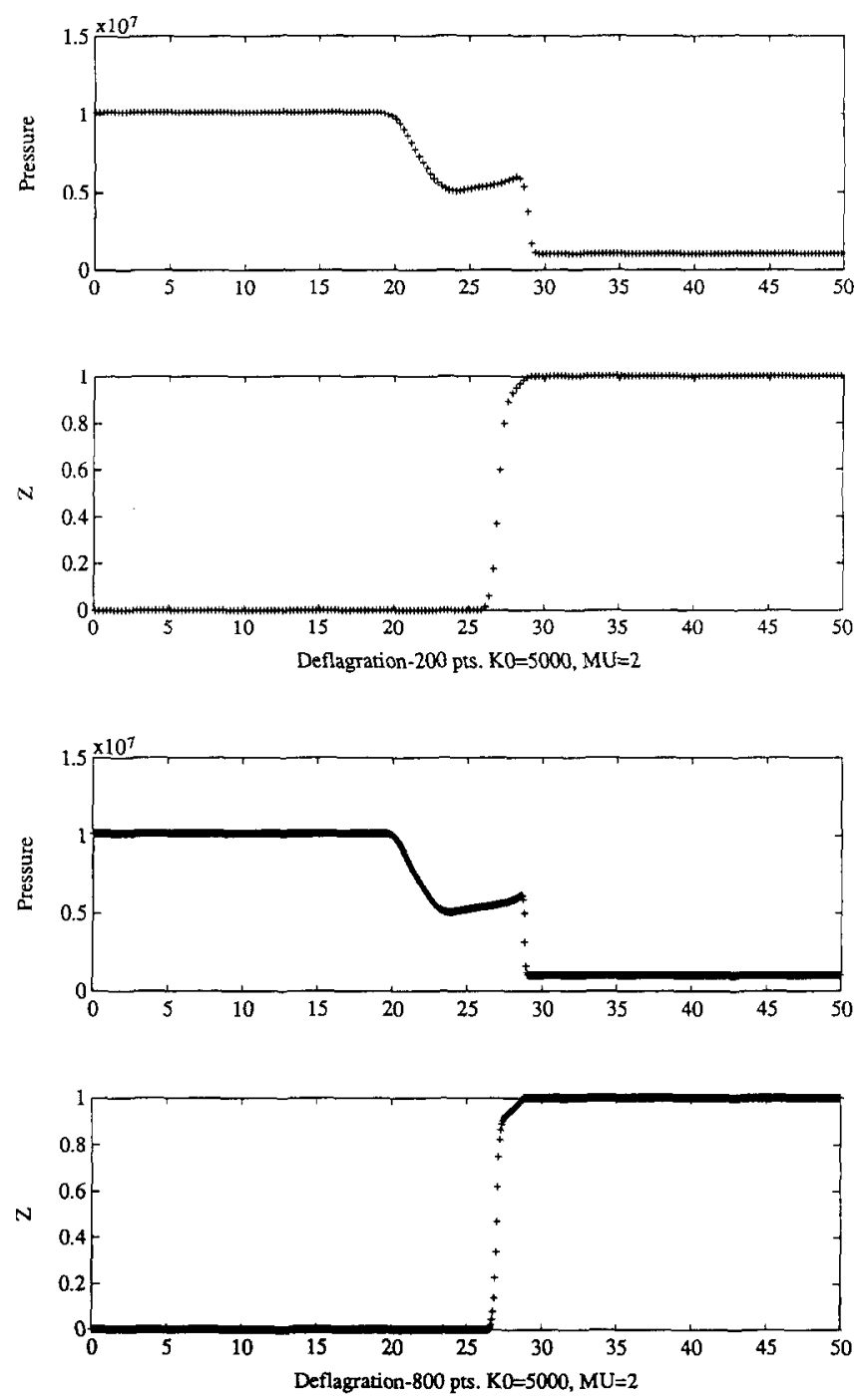

FIG. 1. A deflagration represented by the jump in $Z$ trailing the pressure shock wave. 
is obtained directly from advancing in time in the other equations.

The problem we chose to compute is a Riemann problem, where the initial data is given by

$$
\begin{aligned}
& (\rho, \rho u, \rho E, \rho Z)(x) \\
& \quad= \begin{cases}(0.0016,0,25378756,0) & \text { if } x<0 \\
(0.0012,0,52774715,0.0012) & \text { if } x>0\end{cases}
\end{aligned}
$$

A similar problem has been computed in [3] with standard splitting algorithms. The units are in CGS (centimeters, grams, seconds). We choose the gas constants $c_{v}, c_{p}, \gamma$ to be the appropriate constants for air:

$$
c_{v}=7177250, \quad c_{p}=10048150, \quad \gamma=1.4
$$
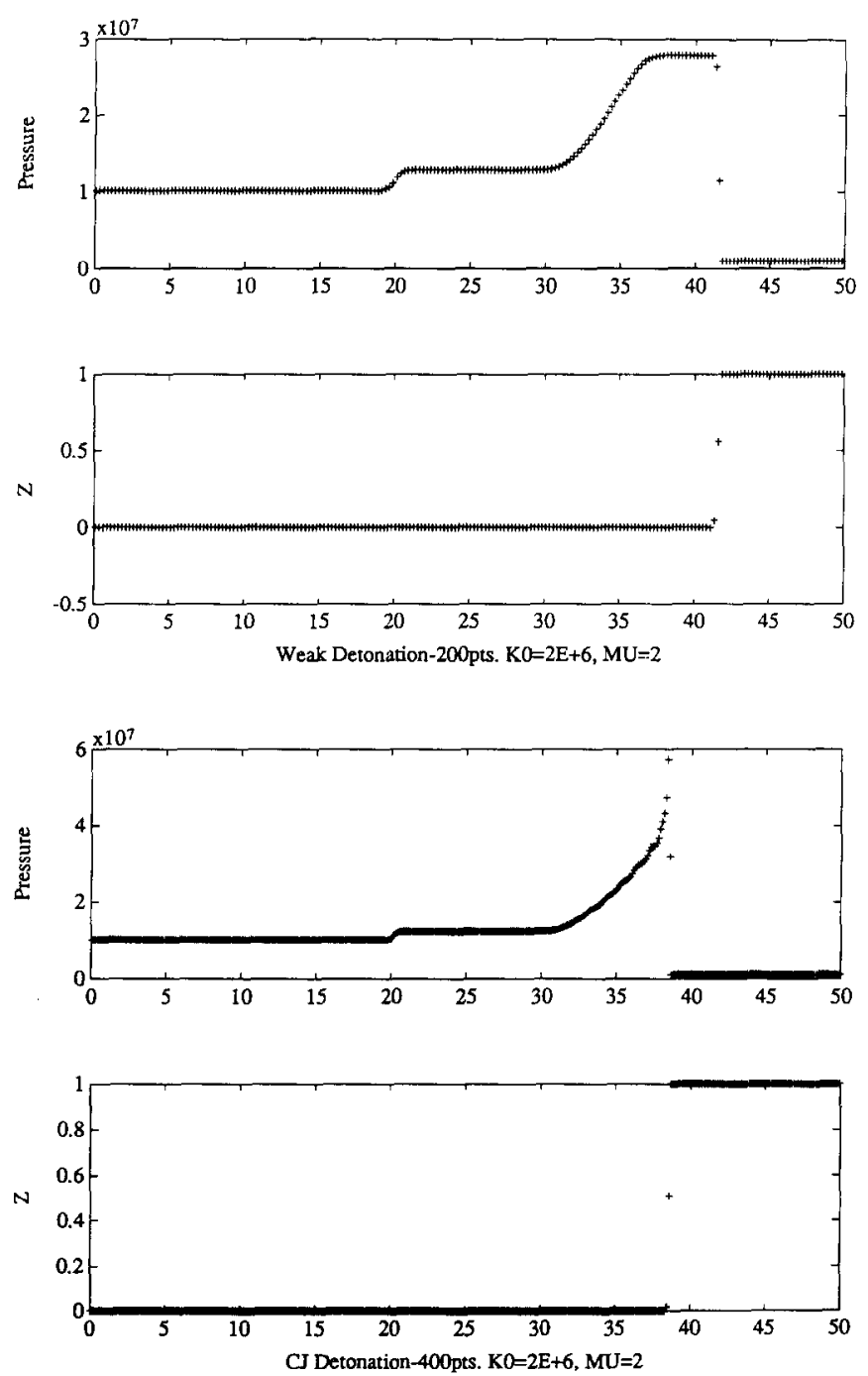

FIG. 2. A non-physical weak detonation computed (top). The physical solution is a $\mathrm{CJ}$ detonation given by the computation with twice the resolution (bottom).
The initial data is such that the fresh gas is on the right, $Z=1$, and the completely burnt gas is on the left, $Z=0$. The fresh gas is at an ambient pressure of $1 \mathrm{~atm}$ and temperature of $294.1 \mathrm{~K}$. We assume that reactions are one-step processes by which a fraction of the fresh gas (for example, a mixture of methane and air) is converted into burnt gas and heat is released. The amount of heat released when the gas is completely burnt is given by $q_{0}$. A reasonable value for $q_{0}$ which is consistent with the type of reaction described is

$$
q_{0}=4.1868 \times 10^{10} \quad(=1 \mathrm{kcal})
$$

(cf. [9]). The completely burnt gas is assumed to be at a temperature of $2210 \mathrm{~K}$ and a pressure of approximately $10 \mathrm{~atm}$. In all our computations the solution is computed at $t=5.5 \times 10^{-5} \mathrm{~s}$ and $T_{*}=500 \mathrm{~K}, \Delta t / \Delta x=$
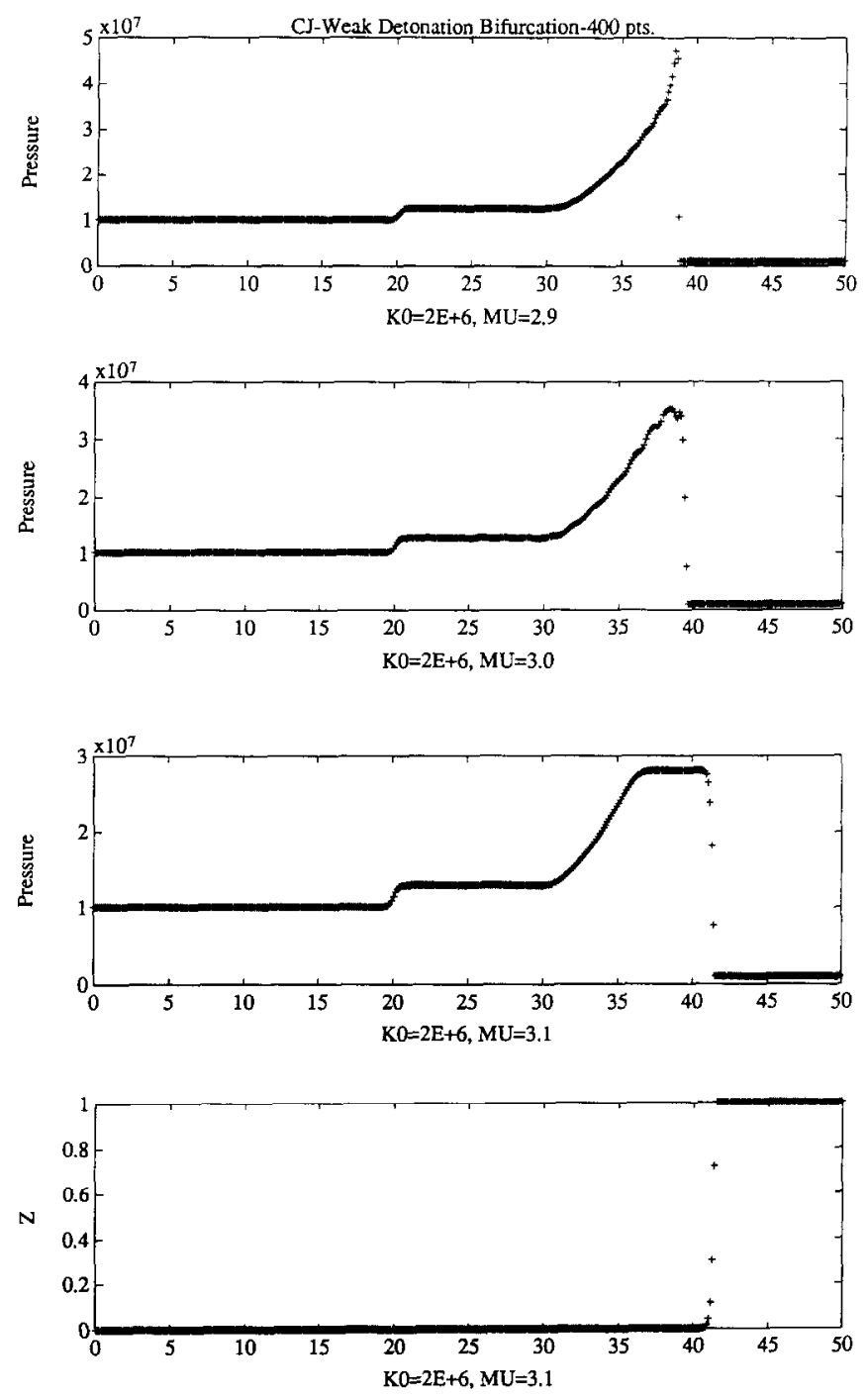

FIG. 3. Bifurcation from a CJ detonation to a weak detonation (top three plots). 
$3 \times 10^{-6}$ (CFL No. $\approx 0.8$ ). The only quantities that change from one computation to another are $K_{0} \Delta t$ and $\Delta x / \mu$.

In Fig. 1 we have computed the constant pressure deflagration which is initiated by a fluid mechanical shock. The top computation is on a coarser mesh $\left(K_{0} \Delta t=0.00375\right.$, $\Delta x / \mu=0.125)$ than the bottom one $\left(K_{0} \Delta t=0.009375\right.$, $\Delta x / \mu=0.03125$ ) if we consider $K_{0}$ and $\mu$ fixed. Both show very good resolution (c.. Fig. 7 in [3], where a comparable solution was computed with $K_{0} \Delta t=4.25 \times 10^{-12}, \Delta x / \mu=$ $6.68 \times 10^{-4}$, i.e., a much finer mesh). In particular, even on the coarse mesh one can distinguish the fluid mechanical shock from the trailing flame.

In Fig. 2 we have raised the value of $K_{0} \Delta t$ to 1.5 (top) and $K_{0} \Delta t=0.75$ (bottom) while keeping the same respective values of $\Delta x / \mu$ as in Fig. 1. We see two completely different wave paterns; the top is a wcak detonation while the bottom
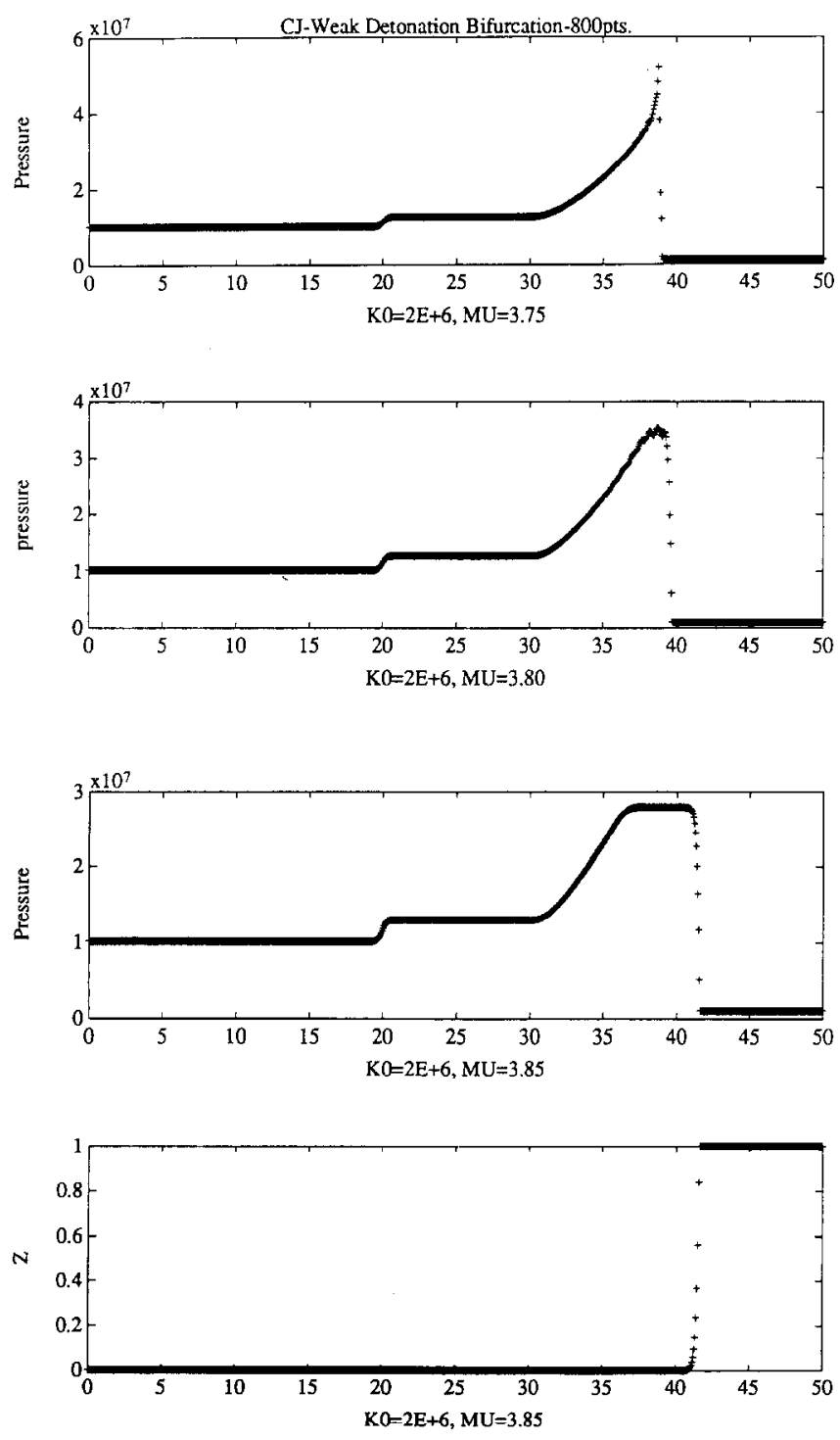

FIG. 4. Same as Fig. 3 with twice the resolution. is a CJ detonation. The top figure is unphysical (for that fixed value of $K_{0}$ ) and it is the result of lack of resolution.

In Fig. 3 we changed the viscosity parameter or $\Delta x / \mu$ while keeping $K_{0} \Delta t$ fixed at 0.75 . There is a bifurcation from a CJ detonation to a weak detonation as $\Delta x / \mu$ is changed from 0.0431 (CJ detonating) to 0.0417 (intermediate ) to 0.0403 (weak detonation). The intermediate solution shows oscillations on a scale much larger than the mesh size. Figure 4 shows the same phenomenon with twice the resolution from Fig. 3, occurring at a slightly different value of $\Delta x / \mu$. As mentioned before, this qualitative behavior was rigorously proved for a $2 \times 2$ model by A. Majda [18].

\section{CONCLUSION}

In this paper we developed a subcell resolution method for viscous perturbations of hyperbolic conservation laws with the hope of getting higher resolution on coarse meshes. This method was tested on computing a problem in combustion and was shown to yield a good resolution of the flow on relatively coarse meshes.

\section{APPENDIX}

In this Appendix we solve for $\phi_{k}$ in (3) (Section 2), using the weakly nonlinear geometrical optics asymptotics. If we substitute (3) into (1) we obtain after cancellation

$$
A_{0}^{\prime} u_{1} u_{1_{x}}=-2 \varepsilon B_{0} u_{1_{x x}}+\text { lower order terms in } u_{1} \text {, }
$$

where $B_{0}=B\left(u_{0}\right)$ and $\left(A_{0}^{\prime} v w\right)_{i}=\sum_{k}\left(\partial a_{0_{i j}} / \partial u_{k}\right) v_{k} w_{j}$.

If from now on we ignore the lower order terms in the equations, which is justified if $u_{1}$ is small, and we substitute the expression of $u_{1}$ from ( 3 ) we obtain

$$
\sum_{k, s} b_{k} b_{s}^{2} \phi_{k} \phi_{s}^{\prime} A_{0}^{\prime} R_{k} R_{s}=-2 \sum_{k} b_{k}^{3} \phi_{k}^{\prime \prime} B_{0} R_{k} .
$$

Multiplying on the left by $L_{l}$, the left eigenvector of $A_{0}$, we obtain

$$
\sum_{k, s} c_{l k s} b_{k} b_{s}^{2} \phi_{k} \phi_{s}^{\prime}=-2 \sum_{k} d_{l k} b_{k}^{3} \phi_{k}^{\prime \prime}, \quad l=1, \ldots, n,
$$

where $\quad c_{l k s}=L_{l} A_{0}^{\prime} R_{k} R_{s}, \quad d_{l k}=L_{l} B_{0} R_{k}, \quad$ and $\quad \phi_{k}=$ $\phi_{k}\left(\left(x-\lambda_{k} t\right) / \varepsilon / b_{k}\right)$ and $\phi_{s}=\phi_{s}\left(\left(x-\lambda_{s} t\right) / \varepsilon / b_{s}\right)$.

Let us define the variables $x_{l}=\left(x-\lambda_{l} t\right) / \varepsilon / b_{l}, l=1, \ldots, n$. Suppose we fix $l$ and consider Eq. (23) evaluated in terms of 
the variable $x_{l}$. Then $x_{k}=\left(b_{k} / b_{l}\right) x_{l}+\left(\lambda_{l}-\lambda_{k}\right) t / \varepsilon / b_{k}=$ $x_{k}\left(x_{l}, t\right)$ and we obtain

$$
\begin{aligned}
& c_{l l l} b_{l}^{3} \phi_{l} \phi_{l}^{\prime}\left(x_{l}\right)+\sum_{k \neq l, s \neq l} c_{l k s} b_{k} b_{s}^{2} \phi_{k}\left(x_{k}\right) \phi_{s}^{\prime}\left(x_{s}\right) \\
& \quad+b_{l} \phi_{l}\left(x_{l}\right) \sum_{s \neq l} c_{l l s} b_{s}^{2} \phi_{s}^{\prime}\left(x_{s}\right) \\
& \quad+b_{l} \phi_{l}^{\prime}\left(x_{l}\right) \sum_{k \neq l} c_{l k l} b_{k}^{2} \phi_{k}\left(x_{k}\right)+2 d_{l l} b_{l}^{3} \phi_{l}^{\prime \prime}\left(x_{l}\right) \\
& \quad+2 \sum_{k \neq l} d_{l k} b_{k}^{3} \phi_{k}^{\prime \prime}\left(x_{k}\right)=0
\end{aligned}
$$

which holds for all $t$ with $x_{l}$ fixed. We follow the procedure in [19] and average every term in the equation with respect to $t$. If we assume that the $\phi_{k}$ 's tend to bounded values at infinity, then several terms are zero and we obtain

$$
\begin{aligned}
& c_{l l l} b_{l}^{3} \phi_{l} \phi_{l}^{\prime}\left(x_{l}\right)+\sum_{k, s \neq l, k \neq s} c_{l k s} b_{k} b_{s}^{2} \lim _{T \rightarrow \infty} \frac{1}{2 T} \\
& \quad \times \int_{-T}^{T} \phi_{k}\left(x_{k}\left(x_{l}, t\right)\right) \phi_{s}^{\prime}\left(x_{s}\left(x_{l}, t\right)\right) d t \\
& \quad+b_{l} \phi_{l}^{\prime}\left(x_{l}\right) \sum_{k \neq l} c_{l k l} b_{k}^{2} \lim _{T \rightarrow \infty} \frac{1}{2 T} \\
& \quad \times \int_{-T}^{T} \phi_{k}\left(x_{k}\left(x_{l}, t\right)\right) d t+2 d_{l l} b_{l}^{3} \phi_{l}^{\prime \prime}\left(x_{l}\right)=0 .
\end{aligned}
$$

If $d_{l l}=L_{l} B_{0} R_{l} \neq 0$ and $c_{l l l}=L_{l} A_{0}^{\prime} R_{l} R_{l}=\nabla \lambda_{l} \cdot R_{l} \neq 0$ (if the field is genuinely nonlinear [17]), we can choose the right eigenvectors $R_{l}$ so that $c_{l l l}=d_{l l}$. Then

$$
\phi_{l}\left(x_{l}\right)=\frac{1}{\alpha_{l}} \tanh \left(\frac{x_{l}-x_{j+1 / 2} b_{l} / \varepsilon}{4 \alpha_{l}}\right), \quad l=1, \ldots, n,
$$

is a solution to the equation above for any $\alpha$. In fact (24) solves the equation $\phi_{l} \phi_{l}^{\prime}+2 \phi_{l}^{\prime \prime}=0$, so we need to verify that the terms involving the $\lim _{T \rightarrow \infty}$ are zero. But this follows from

$$
\begin{aligned}
\frac{1}{2 T} \int_{-T}^{T} \phi_{k}\left(x_{k}\left(x_{l}, t\right)\right) \phi_{s}^{\prime}\left(x_{s}\left(x_{l}, t\right)\right) d t & \\
\leqslant & \frac{1}{\sqrt{2 T}}\left(\frac{1}{2 T} \int_{-T}^{T} \phi_{k}^{2}\left(x_{k}\left(x_{l}, t\right)\right)\right)^{1 / 2} \\
& \times\left(\int_{-I}^{T} \phi_{s}^{\prime 2}\left(x_{s}\left(x_{l}, t\right)\right) d t\right)^{1 / 2}
\end{aligned}
$$

and the fact that (24) implies $\left|\phi_{s}^{\prime}\right|_{L_{2}}$,

$$
\left((1 / 2 T) \int_{-T}^{T} \phi_{k}^{2}\left(x_{k}\left(x_{l}, t\right)\right) d t\right)^{1 / 2} \leqslant \infty
$$

and that

$$
\lim _{T \rightarrow \infty} \frac{1}{2 T} \int_{-T}^{T} \phi_{k}\left(x_{k}\left(x_{l}, t\right)\right) d t=0 .
$$

Remarks. The terms involving the $\lim _{T \rightarrow \infty}$ which couple the different $\phi$ 's and which vanish in our case are called resonances [19]. We do not have a proof that (24) is the only solution with that boundary behavior at $\infty$ but we believe it to be true. The subcell approximation is obtained by substituting (24) into (3) (Section 2):

$$
\begin{aligned}
u(x, t)= & u_{0}-\frac{1}{2} \sum_{k} b_{k} \frac{1}{\alpha_{k}} \\
& \times \tanh \left(\frac{x-x_{j+1 / 2}-\lambda_{k} t}{4 \alpha_{k} \varepsilon / b_{k}}\right) R_{k}, \quad x \in I_{j} .
\end{aligned}
$$

\section{REFERENCES}

1. A. Bourlioux, A. Majda, and V. Roytburd, Theoretical and numerical structure for unstable one-dimensional detonations, preprint (1989).

2. A. J. Chorin, J. Comput. Phys. 25 (1977).

3. P. Collela, A. Majda, and V. Roytburd, SIAM J. Stat. Comput. 7, No. 4 (1986).

4. Courant and Friedrichs, Supersonic Flow and Shock Waves, SpringerVerlag, New York/Berlin, 1976.

5. Courant and Hilbert, Methods in Mathematical Physics II, Interscience, New York, 1962.

6. J. D Buckmaster and G. S. S. Ludford, Lectures on Mathematical Combustion, CBMS-NSF Regional Conf. Series in Appl. Math., 1983.

7. R. DiPerna and A. Majda, Commun. Math. Phys. k98, No. 3 (1985).

8. B. Engquist and B. Sjogreen, Robust Difference Approximations of Stiff Inviscid Detonation Waves, UCLA CAM Report 91-03, 1991.

9. I. Glassman, Combustion, 2 ed., Academic Press, New York, 1987.

10. E. Harabetian, SIAM J. Numer. Anal. 27 (1990)

11. E. Harabetian, A Numerical Method for Computing Viscous Shock Layers, Notes on Numer. Fluid Mechanics, edited by Josef Ballman and Rolf Jeltsch, Vol. 24, p. 220.

12. A. Harten, ENO Schemes with Subcell Resolution, UCLA Comput. and Appl. Math. Report 87-13, 1987.

13. A. Harten, J. Comput. Phys. 49 (1983).

14. J. Hunter and J. Keller, Commun. Pure Appl. Math. 36 (1983).

15. S. N. Kružkov, Math. USSR-Sb. 27 (1975).

16. O. A. Ladyženskaja, V. A. Solonnikov, and N. N. Ural'ceva, Linear and Quailinear Equations of Parabolic type, Amer. Math. Soc., Providence, RI, 1968

17. P. D. Lax, Hyperbolic Systems of Conservation Laws and the Mathematical Theory of Shock Waves, SIAM Reg. Conf. Ser. in Appl. Math., Vol. 11, SIAM, Philadelphia, 1973.

18. A. Majda, SIAM J. Appl. Math. 41 (1981).

19. A. Majda and R. Rosales, Stud. Appl. Math. 71, 149 (1984).

20. S. Osher, SIAM J. Numer. Anal. 21 (1984).

21. P. Roe, J. Comput. Phys. 43 (1981).

22. J. Smoller, Shock Waves and Reaction Diffusion Equations, SpringerVerlag, New York/Bcrlin, 1983.

23. P. K. Sweby, SIAM J. Numer. Anal. 21 (1984). 